\title{
ON COMPLEMENTATION OF VECTOR-VALUED HARDY SPACES
}

\author{
WOLFGANG HENSGEN
}

(Communicated by Paul S. Muhly)

\begin{abstract}
Let $X$ be a complex Banach space and $1<p<\infty$. $H^{p}(X)$ resp. $h^{p}(X)$ denote the Hardy spaces of $X$-valued analytic resp. harmonic functions on the disc. $L^{p}(X)$ is the Lebesgue-Bochner space of $X$-valued integrable functions on the circle and $\mathbf{H}^{p}(X)$ its Hardy-type subspace $\left\{f \in L^{p}(X): \hat{f}(n)=\right.$ $0 \forall n<0\}$. It is proved that the following four conditions are equivalent: $H^{p}(X)$ is complemented in $h^{p}(X)$; the canonical analytic (or Riesz) projection is a bounded operator $h^{p}(X) \rightarrow H^{p}(X) ; \mathbf{H}^{p}(X)$ is complemented in $L^{p}(X)$; analytic projection is a bounded operator $L^{p}(X) \rightarrow \mathbf{H}^{p}(X)$. It is well known that the last condition, in turn, is equivalent to the UMD property of $X$.
\end{abstract}

1. Introduction and statement of result. For $1 \leq p \leq \infty$, let $L^{p}(T, \lambda)$ be the Lebesgue space on the unit circle $T$ with normalized Lebesgue measure $\lambda$, and let $H^{p} \subset L^{p}$ be the usual Hardy space (so $H^{p}=\left\{f \in L^{p}: \hat{f}(n)=0 \forall n<0\right\}$, where $\hat{f}(n)=\int_{0}^{2 \pi} e^{-i n t} f\left(e^{i t}\right) \frac{d t}{2 \pi}$ is the $n$th Fourier coefficient of $\left.f ; n \in \mathbf{Z}\right)$. The famous M. Riesz theorem [15, p. 151ff.] states that for $1<p<\infty, H^{p}$ is complemented in $L^{p}$ by the canonical "analytic (or Riesz) projection" $f \mapsto f^{a}$, where $f^{a}$ is that $L^{p}$ function with Fourier series $\sum_{n=0}^{\infty} \hat{f}(n) e^{i n t}$. For $p=1$ or $p=\infty, f \mapsto f^{a}$ is not a bounded operator in $\|\cdot\|_{p}$ (on the trigonometric polynomials, say). Actually, by Newman's theorem, $H^{1}$ is not complemented in $L^{1}$ at all, and the same is true for $H^{\infty}$ in $L^{\infty}[15$, p. 154ff.]. There is a beautiful proof of Newman's theorem due to Rudin. Quoting Hoffman [15, p. 154ff.], "the idea of the proof is to show that if there is any bounded projection of $L^{1}$ onto $H^{1}$ then the 'natural' projection must be bounded.... The proof...works equally well for projections from $L^{p}$ to $H^{p}$ as long as $1 \leq p<\infty$; that is, the proof shows that if there is any bounded projection from $L^{p}$ to $H^{p}$, then the natural projection is bounded." The purpose of this article is to show that this "principle" remains true in a Banach space valued setting. It is perhaps worth noting that Rudin's proof uses Banach space valued integration although the theorem deals exclusively with scalar functions.

Let $Y$ be a complex Banach space and $L^{p}(Y)=L^{p}(\lambda ; Y)$ be the LebesgueBochner space which is assumed to be familiar to the reader [7, II]. In view of $H^{p}$ 's definition above, one natural $Y$-valued $H^{p}$ space is $\mathbf{H}^{p}(Y):=\left\{f \in L^{p}(Y): \hat{f}(n)=\right.$ $0 \forall n<0\} \subset L^{p}(Y)$, where again $\hat{f}(n)=\int_{0}^{2 \pi} e^{-i n t} f(t) \frac{d t}{2 \pi} \in Y$ is the $n$th Fourier coefficient of $f ; n \in \mathbf{Z}$. It is part of the result of this paper (stated at the end of

Received by the editors December 9, 1987.

1980 Mathematics Subject Classification (1985 Revision). Primary 46E40, 42A50; Secondary 46J15, 46B20.

Key words and phrases. Vector-valued Hardy spaces, analytic (or Riesz) projection, UMD Banach spaces. 
$\S 1$ ) that $\mathbf{H}^{p}(Y)$ is complemented in $L^{p}(Y)$ (if and) only if it is so by the natural analytic projection; the proof of this fact alone would be a verbatim copy of Rudin's proof in [15, p. 154].

On the other hand, the most classical definition of $H^{p}$ is, of course, $H^{p}=$ $\left\{f: D \rightarrow \mathbf{C}\right.$ analytic: $\left.\|f\|_{p}<\infty\right\}$, where $D$ is the unit disc and

$$
\|f\|_{p}^{p}=\sup _{r<1} \int_{0}^{2 \pi}\left|f\left(r e^{i t}\right)\right|^{p} \frac{d t}{2 \pi} \quad(p<\infty)
$$

resp. $\|f\|_{\infty}=\sup _{z \in D}|f(z)|$. (As is well known, the two $H^{p}$ spaces are isometric via Poisson integral resp. boundary values [15, Chapters 3 and 4].) This holomorphic definition also easily generalizes rendering the $Y$-valued $H^{p}$ space $H^{p}(Y)=\left\{f: D \rightarrow Y\right.$ analytic: $\left.\|f\|_{p}<\infty\right\}$, where

$$
\|f\|_{p}^{p}=\sup _{r<1} \int_{0}^{2 \pi}\left\|f\left(r e^{i t}\right)\right\|_{Y}^{p} \frac{d t}{2 \pi} \quad(p<\infty)
$$

resp. $\|f\|_{\infty}=\sup _{z \in D}\|f(z)\|_{Y}$. Now Poisson integral, in general, embeds $\mathbf{H}^{p}(Y)$ isometrically only as a closed subspace into $H^{p}(Y)$, and an $H^{p}(Y)$ function need not possess any radial limits (not even weak* in $Y^{\prime \prime}[10$ ex. 1, 13, (2.17), (3.3)]). Nevertheless, there is a Banach space $L_{Y}^{p}\left(X^{\prime}, X\right)$ of Gel'fand integrable $X^{\prime}$-valued functions, where $X$ is any Banach space such that $Y \subset X^{\prime}$, with the property that every $f \in H^{p}(Y)$ is the Poisson(-Gel'fand) integral of a unique $\varphi \in L_{Y}^{p}\left(X^{\prime}, X\right)$. This identifies $H^{p}(Y)$ isometrically with the subspace

$$
L_{Y, a}^{p}\left(X^{\prime}, X\right):=\left\{\varphi \in L_{Y}^{p}\left(X^{\prime}, X\right): \hat{\varphi}(n)=0 \forall n<0\right\}
$$

of $L_{Y}^{p}\left(X^{\prime}, X\right)$, completely analogous to the inclusion $\mathbf{H}^{p}(Y) \subset L^{p}(Y)$. See $\S 2$ for details. The main assertion now is that also $H^{p}(Y)$ is complemented in $L_{Y}^{p}\left(X^{\prime}, X\right)$ (if and) only if it is so by the natural analytic projection. The proof, again, follows Rudin's idea but is technically more complicated since it has to use the Gel'fand instead of the Bochner integral. Moreover, the two complementation assertions for $\mathbf{H}^{p}(Y)$ resp. $H^{p}(Y)$ are equivalent.

Before writing down the theorem completely, I want to eliminate the auxiliary space $X$ and the artificially looking $L_{Y}^{p}\left(X^{\prime}, X\right)$. In fact via Poisson integral $L_{Y}^{p}\left(X^{\prime}, X\right) \cong h^{p}(Y):=\left\{f: D \rightarrow Y\right.$ harmonic: $\left.\|f\|_{p}<\infty\right\}$ for $1<p \leq \infty$, where $\|f\|_{p}$ is defined as above; the inclusion $L_{Y, a}^{p}\left(X^{\prime}, X\right) \subset L_{Y}^{p}\left(X^{\prime}, X\right)$ just translates into the natural $H^{p}(Y) \subset h^{p}(Y)$, and the analytic projection of an $h^{p}(Y)$ function $f\left(r e^{i t}\right)=\sum_{n=-\infty}^{\infty} y_{n} r^{|n|} e^{i n t}$ is $f^{a}(z)=\sum_{n=0}^{\infty} y_{n} z^{n}$. The details are given in $\S 2$. We are thus led to the following final formulation of the

THEOREM. Let $1<p<\infty$ and $Y$ a Banach space. The following four assertions are equivalent.

$\left(1^{\circ}\right)$ (a) The analytic projection $f \mapsto f^{a}$ is a bounded operator $L^{p}(Y) \rightarrow \mathbf{H}^{p}(Y)$.

(b) The analytic projection $f \mapsto f^{a}$ is a bounded operator $h^{p}(Y) \rightarrow H^{p}(Y)$.

$\left(2^{\circ}\right)(\mathrm{a}) \mathbf{H}^{p}(Y)$ is complemented in $L^{p}(Y)$.

(b) $H^{p}(Y)$ is complemented in $h^{p}(Y)$.

The extreme values $p=1, \infty$ have been excluded since, modulo scalar theory, the situation is trivial in these cases, cf. Remark 3.3. 
For dual spaces $Y$, the equivalence of $\left(1^{\circ}\right)(\mathrm{a})$ and (b) is contained in [3, Theorem $3.1]$. It is part of the folklore $([3$, p. 1057,5 , Lemmas 3 and 4,6, p. 66, 11]; see $[13, \S 5]$ for a detailed exposition) that condition $\left(1^{\circ}\right)(\mathrm{a})$ is equivalent to the $\|\cdot\|_{p}$ boundedness of the $Y$-valued Hilbert transform. By the work of Burkholder [5, 6] and Bourgain [1] this, in turn, is equivalent to the probabilistic "UMD" condition on $Y$.

This work is part of my doctoral thesis [13] written at the University of Munich.

2. Preliminaries on vector-valued Hardy spaces. For a detailed discussion of the following, cf. $[13, \S \S 0,1,2$ and 5]. I repeat that the basic theory of the Bochner integral and Bochner-Lebesgue spaces $L^{p}(X)=L^{p}(\lambda ; X)$ is supposed to be known [7, II]. I am going to explain the less familiar notion of Gel'fand integral and the spaces $L^{p}\left(X^{\prime}, X\right)$ first. In what follows, $X, Y$ are (complex) Banach spaces, $X^{\prime}$ denotes the dual of $X$, and $1 \leq p, q \leq \infty$.

2.1 Gel fand integral; spaces $L^{p}\left(X^{\prime}, X\right)$. A function $f: T \rightarrow X^{\prime}$ is called scalarly integrable (w.r.t. $X$ ) if the function $\langle x, f\rangle: T \rightarrow \mathbf{C}$ is integrable for all $x \in X$. In this case, for any Borel set $E \subset T$, the Gel'fand integral $(G) \int_{E} f d \lambda \in X^{\prime}$ is well defined by the formula $\left\langle x,(G) \int_{E} f d \lambda\right\rangle:=\int_{E}\langle x, f\rangle d \lambda, x \in X[\mathbf{7}, \mathrm{p}$. 53]. The symbol $(G)$ will often be suppressed.

Now recall that the Banach lattice $L^{p}(\lambda ; \mathbf{R})$ is order complete $[18$, p. 4], i.e. every order bounded subset of $L^{p}$ has a supremum in $L^{p}$ (supremum in the sense of order in $L^{p}$, denoted by $L^{p}$-sup). Put

$$
\begin{aligned}
& \mathscr{L}^{p}\left(X^{\prime}, X\right)=\mathscr{L}^{p}\left(\lambda ; X^{\prime}, X\right):=\left\{f: T \rightarrow X^{\prime}:\langle x, f\rangle \in L^{p} \quad \forall x \in X\right. \\
&\text { and } \left.\{\langle x, f\rangle:\|x\| \leq 1\} \text { is order bounded in } L^{p}\right\} .
\end{aligned}
$$

Following Bukhvalov and Danilevich $[\mathbf{3}, 0 ., 4,1$.$] , one defines for f \in \mathscr{L}^{p}\left(X^{\prime}, X\right)$ the $L^{p}$ function $v_{f}:=L^{p}-\sup _{\|x\| \leq 1}|\langle x, f\rangle|$ and the seminorm $\|f\|_{p}:=\left\|v_{f}\right\|_{p}$. The null space of the seminorm $\|\cdot\|_{p}$ on $\mathscr{L}^{p}\left(X^{\prime}, X\right)$ is easily recognized as $\{f \in$ $\mathscr{L}^{p}\left(X^{\prime}, X\right): \quad \forall x \in X:\langle x, f\rangle=0$ a.e. $\}$. (Note that in the formulation " $\forall x \in$ $X:\langle x, f\rangle=0$ a.e." the exceptional null set depends on $x$. Typical example: $f: T \rightarrow$ $l^{2}(T), f(t):=e_{t}$, the $t$ th unit vector. We have $\|f(\cdot)\|=1$, but $\|f\|_{p}=0$.)

Finally, put $L^{p}\left(X^{\prime}, X\right)=L^{p}\left(\lambda ; X^{\prime}, X\right):=\mathscr{L}^{p}\left(X^{\prime}, X\right) /\|\cdot\|_{p}^{-1}(0)$ with the associated norm $\|\varphi\|_{p}=\|f\|_{p}\left(f \in \varphi \in L^{p}\left(X^{\prime}, X\right)\right)$. For $\varphi \in L^{p}\left(X^{\prime}, X\right),\langle x, \varphi\rangle \in$ $L^{p}(x \in X)$ and $(G) \int_{E} \varphi d \lambda(E \subset T$ Borel $)$ are well defined, since independent of the choice of representative. Obviously, we have $L^{p}\left(X^{\prime}\right) \subset L^{p}\left(X^{\prime}, X\right)$ as a closed subspace (i.e., the canonical map is an isometry, cf. e.g. $\left.\left[13,(0.5) 4^{\circ}\right]\right)$.

Now let $1 / p+1 / q=1, f \in L^{p}(X), g \in L^{q}\left(X^{\prime}, X\right)$. It is not hard to see $\left[13,(0.5) 5^{\circ}\right]$ that $\langle f, g\rangle: T \rightarrow \mathbf{C}$ is a (well-defined!) member of $L^{1}$ satisfying $\int_{T}|\langle f, g\rangle| d \lambda \leq\|f\|_{p}\|g\|_{q}$ so that $g$ acts as a bounded linear functional of norm at most $\|g\|_{q}$ on $L^{p}(X)$. The importance of the space $L^{q}\left(X^{\prime}, X\right)$ lies in the fact that it is the exact dual space of $L^{p}(X)(1 \leq p<\infty)$ :

THEOREM. Let $X$ be a Banach space, $1 \leq p<\infty, 1 / p+1 / q=1$. The map

$$
L^{q}\left(X^{\prime}, X\right) \rightarrow L^{p}(X)^{\prime}, \quad g \mapsto \int_{T}\langle\cdot, g\rangle d \lambda
$$

is a (well-defined) isometric isomorphism. ("Isomorphism" is meant to include surjectivity.) 
This description of $L^{p}(X)^{\prime}$ is due to Bukhvalov [2, Theorem 7, 3, Theorem 0.1]. It is in a canonical sense equivalent $[\mathbf{1 3},(0.7)]$ to the perhaps more widespread representation of $L^{p}(X)^{\prime}$ due to Ionescu-Tulcea [16, VII Theorem 7 Corollary, Theorem 9 Corollary].

2.2 Poisson integral; spaces $L_{Y}^{p}\left(X^{\prime}, X\right)$. For $\varphi \in L^{p}\left(X^{\prime}, X\right)$, we denote by $\hat{\varphi}(n)=$ $\int_{0}^{2 \pi} e^{-i n t} \varphi(t) \frac{d t}{2 \pi} \in X^{\prime}$ the $n$th Fourier coefficient $(n \in \mathbf{Z})$ and by $P[\varphi]: D \rightarrow X^{\prime}$, $P[\varphi]\left(r e^{i \theta}\right):=\int_{0}^{2 \pi} P_{r}(\theta-t) \varphi(t) \frac{d t}{2 \pi}$ the Poisson integral of $\varphi$. (The integrals are, of course, Gel'fand integrals.) Here $P_{r}$ is the Poisson kernel, $P_{r}(t)=\left(1-r^{2}\right) /(1-$ $\left.2 r \cos t+r^{2}\right)$. An easy computation yields, as in the scalar case, $P[\varphi]\left(r e^{i \theta}\right)=$ $\sum_{n=-\infty}^{\infty} \hat{\varphi}(n) r^{|n|} e^{i n \theta}$ with absolutely and in $D$ locally uniformly convergent series.

Now let $Y \subset X^{\prime}$ be a Banach subspace. The following conditions on $\varphi \in$ $L^{p}\left(X^{\prime}, X\right)$ are equivalent [13, Lemma (1.5)]:

$$
\begin{gathered}
\int_{E} \varphi d \lambda \in Y \quad \forall E \subset T \text { Borel; } \quad \int_{T} g f d \lambda \in Y \quad \forall g \in L^{q}\left(\frac{1}{p}+\frac{1}{q}=1\right) ; \\
\hat{\varphi}(n) \in Y \quad \forall n \in \mathbf{Z} ; \quad P[\varphi](z) \in Y \quad \forall z \in D .
\end{gathered}
$$

The space of those $\varphi \in L^{p}\left(X^{\prime}, X\right)$ is denoted by $L_{Y}^{p}\left(X^{\prime}, X\right)$; obviously $L^{p}(Y) \subset$ $L_{Y}^{p}\left(X^{\prime}, X\right) \subset L^{p}\left(X^{\prime}, X\right)$ as closed subspaces.

2.3 Vector-valued $h^{p}$-spaces. We define $h^{p}(Y):=\left\{u: D \rightarrow Y\right.$ harmonic: $\|u\|_{p}<$ $\infty\}$ where $\|u\|_{p}=\sup _{r<1}\left\|u_{r}\right\|_{p}$ and $u_{r}: T \rightarrow Y ; u_{r}\left(e^{i t}\right):=u\left(r e^{i t}\right)$. A word on the notion of a Banach space valued harmonic function seems in order. Exactly as in the better known case of holomorphic functions [14, Chapter III.2] any two reasonable definitions of harmonicity for a Banach space valued function are equivalent. To be more specific, any of the following conditions on $u: D \rightarrow Y$ implies all the others [13 (1.3)]:

(i) $u$ is strongly harmonic, i.e. $u \in C^{2}(D, Y)$ and $\Delta u=0$.

(ii) $u$ is weakly harmonic, i.e. $\left\langle u, y^{\prime}\right\rangle$ is harmonic $\forall y^{\prime} \in Y^{\prime}$.

(iii) (If $Y=X^{\prime}$ ) $u$ is weak ${ }^{*}$ harmonic, i.e. $\langle x, u\rangle$ is harmonic $\forall x \in X$.

(iv) $\exists y_{n} \in Y(n \in \mathbf{Z})$ such that $u\left(r e^{i \theta}\right)=\sum_{n=-\infty}^{\infty} y_{n} r^{|n|} e^{i n \vartheta}$ in $D$ with absolutely and locally uniformly convergent series.

The following Poisson integral representation theorem [13, Theorem (1.5)] is essentially a concise formulation of results of Grossetête $[\mathbf{9}, \S 1]$ and Bukhvalov [3, Theorem 2.3]. Let $Y \subset X^{\prime}$ as in 2.2 (e.g., $X:=Y^{\prime}$ ).

THEOREM. The Poisson integral defines

$\left(1^{\circ}\right)$ an isometry $P: L_{Y}^{1}\left(X^{\prime}, X\right) \rightarrow h^{1}(Y), \varphi \mapsto P[\varphi]$,

$\left(2^{\circ}\right)$ an isometric isomorphism $P: L_{Y}^{p}\left(X^{\prime}, X\right) \rightarrow h^{p}(Y), \varphi \mapsto P[\varphi]$ if $1<p \leq \infty$.

For the sake of clarity, I remark that the isometry in $\left(1^{\circ}\right)$ is never surjective (except $Y=0$ ). The full representation space for $h^{1}(Y)$ would be $M(Y)$, the space of $Y$-valued ( $\sigma$-additive) vector measures with bounded variation on the Borel sets of $T[\mathbf{9}, \S 1, \mathbf{1 2}$, Theorem 3.1, 13, Theorem (1.5), 19, Theorem 2.3]. The space $L_{Y}^{1}\left(X^{\prime}, X\right)$ appearing above corresponds via the identification $\varphi \mapsto \varphi d \lambda$ exactly to the subspace $M_{c}(Y)$ consisting of $\lambda$-absolutely continuous members of $M(Y)$ ([13, Theorem $\left.(0.8) 4^{\circ}\right]$; this is essentially the "generalized theorem of LebesgueNikodým" [8, §13, Theorem 5]).

2.4 Vector-valued $H^{p}$ spaces; spaces $L_{Y, a}^{p}\left(X^{\prime}, X\right)$. We define $H^{p}(Y):=\{f \in$ $h^{p}(Y): f$ is analytic $\}$. See [14, Chapter III.2] for the notion of analyticity of a 
Banach space valued function. Let again $Y \subset X^{\prime}$ as in 2.2 and define $L_{Y, a}^{p}\left(X^{\prime}, X\right):=$ $\left\{\varphi \in L_{Y}^{p}\left(X^{\prime}, X\right): \hat{\varphi}(n)=0 \quad \forall n<0\right\}$, the subspace of "analytic" members of $L_{Y}^{p}\left(X^{\prime}, X\right)$. By the series expansion of $P[\varphi]$ given in 2.2 it is obvious that for $\varphi \in L_{Y}^{p}\left(X^{\prime}, X\right), \varphi \in L_{Y, a}^{p}\left(X^{\prime}, X\right)$ iff $P[\varphi]$ is analytic. Therefore, at least if $p>1$, the following Poisson integral representation theorem is a trivial consequence of the preceding one. It is due to Ryan [20, Theorems 1 and 3, 21, Theorem 2] in special cases and Bukhvalov [3, Theorem 2.7] in general form. Cf. also [13, Theorem (2.5)].

ThEOREM. For $1 \leq p \leq \infty$, the Poisson integral defines an isometric isomorphism

$$
P: L_{Y, a}^{p}\left(X^{\prime}, X\right) \rightarrow H^{p}(Y), \varphi \mapsto P[\varphi] .
$$

In view of Theorem $2.3\left(1^{\circ}\right)$ and the remarks following it, the theorem for $p=1$ is tantamount to the knowledge that every "analytic" member of $M(Y)$ is already in $M_{c}(Y)$, i.e. the vector-valued F. and M. Riesz theorem [9, 2. Corollary, 13, Theorem (2.3), 17, p. 316, 21, Theorem 1] which in turn is a trivial consequence of the scalar-valued one.

Finally, let $\mathbf{H}^{p}(Y):=\left\{\varphi \in L^{p}(Y): \hat{\varphi}(n)=0 \quad \forall n<0\right\}$ be the space of "analytic" members of $L^{p}(Y)$. In other words, $\mathbf{H}^{p}(Y)=L_{Y, a}^{p}\left(X^{\prime}, X\right) \cap L^{p}(Y)$, intersection in $L_{Y}^{p}\left(X^{\prime}, X\right)$.

2.5 Analytic projection. Let $\varphi \in L^{1}(Y)$. If there exists $\psi \in L^{1}(Y)$ with (formal) Fourier series $\psi \sim \sum_{n=0}^{\infty} \hat{\varphi}(n) e^{i n t}$, this (necessarily unique) $\psi$ is called the "analytic projection" of $\varphi$, denoted by $\varphi^{a}$. Obviously, $\varphi^{a} \in \mathbf{H}^{1}(Y)$ if existent. The precise meaning of condition $\left(1^{\circ}\right)(\mathrm{a})$ in the theorem is thus:

$\left(1^{\circ}\right)(\mathrm{a}) \exists A_{p}<\infty \quad \forall \varphi \in L^{p}(Y) \exists \varphi^{a} \in L^{p}(Y)$ such that

$$
\varphi^{a} \sim \sum_{n=0}^{\infty} \hat{\varphi}(n) e^{i n t} \quad \text { and } \quad\left\|\varphi^{a}\right\|_{p} \leq A_{p}\|\varphi\|_{p} .
$$

On the other hand, since $Y$-valued trigonometric polynomials $\sum_{n=-N}^{N} y_{n} e^{i n t}$ $\left(N \in \mathbf{Z}, y_{n} \in Y\right)$ are dense in $L^{p}(Y), 1 \leq p<\infty[\mathbf{1 3}$, Lemma (3.2)] and $\varphi \mapsto \hat{\varphi}(n)$ is a continuous map $L^{p}(Y) \rightarrow Y,\left(1^{\circ}\right)(\mathrm{a})$ is equivalent to $\|\cdot\|_{p}$ boundedness of $\varphi \mapsto \varphi^{a}$ on the $Y$-valued trigonometric polynomials $\varphi$.

The situation is somewhat easier for harmonic functions on the disc, since there is no existence problem. Let $u: D \rightarrow Y$ be harmonic. By the remarks on harmonicity in $2.3 u$ has an (of course unique) series expansion $u\left(r e^{i \theta}\right)=$ $\sum_{n=-\infty}^{\infty} y_{n} r^{|n|} e^{i n \vartheta}, y_{n} \in Y$, absolutely and locally uniformly convergent in $D$. Then $u^{a}(z):=\sum_{n=0}^{\infty} y_{n} z^{n}$ is plainly also absolutely and locally uniformly convergent in $D$ and thus an analytic function $u^{a}: D \rightarrow Y$, called the analytic projection of $u$. The meaning of condition $\left(1^{\circ}\right)(\mathrm{b})$ in the theorem is now clear.

Finally, observe that these two definitions of analytic projection correspond to each other via Poisson integral. That is, if $\varphi \in L^{1}(Y)$ and $\varphi^{a} \in L^{1}(Y)$ exists, then $P[\varphi]^{a}=P\left[\varphi^{a}\right]$.

3. Proof of theorem. Noting that the implications $\left(1^{\circ}\right)(\mathrm{a}) \Rightarrow\left(2^{\circ}\right)(\mathrm{a})$ and $\left(1^{\circ}\right)(\mathrm{b}) \Rightarrow\left(2^{\circ}\right)(\mathrm{b})$ are trivial, I will first show the equivalence of $\left(1^{\circ}\right)(\mathrm{a})$ and $(\mathrm{b})$. Then I will prove that $\left(2^{\circ}\right)(\mathrm{a})$ or $(\mathrm{b})$ implies $\left(1^{\circ}\right)(\mathrm{a})$. This will establish the asserted equivalence of all four conditions. 
3.1 Proof of $\left(1^{\circ}\right)(\mathrm{a}) \Leftrightarrow(\mathrm{b})$. The implication " $\Leftarrow$ " is almost trivial in view of the remark made in 2.5 , namely that $\left(1^{\circ}\right)$ (a) is equivalent to $\|\cdot\|_{p}$ boundedness of analytic projection of trigonometric polynomials: Let $\varphi$ be a $Y$-valued trigonometric polynomial. By theorem 2.3,

$$
\begin{aligned}
\left\|\varphi^{a}\right\|_{\mathbf{H}^{p}(Y)} & =\left\|P\left[\varphi^{a}\right]\right\|_{H^{p}(Y)}=\left\|P[\varphi]^{a}\right\|_{H^{p}(Y)} \\
& \leq A_{p}\|P[\varphi]\|_{h^{p}(Y)}=A_{p}\|\varphi\|_{L^{p}(Y)}
\end{aligned}
$$

where $A_{p}$ is the norm of the analytic projection in $\left(1^{\circ}\right)(\mathrm{b})$.

Turning to the reverse implication, assume that $\left(1^{\circ}\right)(\mathrm{a})$ is true with norm $A_{p}$, and let $u \in h^{p}(Y), u\left(r e^{i \vartheta}\right)=\sum_{n=-\infty}^{\infty} y_{n} r^{|n|} e^{i n \vartheta}\left(y_{n} \in Y\right.$; cf. 2.3). Fix $R<1$ for the moment, and denote, as in 2.3 , by $u_{R}: T \rightarrow Y$ the (continuous) function $u_{R}\left(e^{i t}\right)=u\left(R e^{i t}\right)$. Plainly, $u_{R} \in L^{p}(Y)$ and $\left\|u_{R}\right\|_{L^{p}(Y)} \leq\|u\|_{h^{p}(Y)}$. Since $u_{R}\left(e^{i t}\right)=\sum_{n=-\infty}^{\infty} y_{n} R^{|n|} e^{i n t}$ converges even uniformly on $T$, the latter series is the Fourier series of $u_{R}$. By assumption, there exists $u_{R}^{a} \in L^{p}(Y),\left\|u_{R}^{a}\right\|_{p} \leq$ $A_{p}\left\|u_{R}\right\|_{p} \leq A_{p}\|u\|_{p}$, with (formal) Fourier series $u_{R}^{a} \sim \sum_{n=0}^{\infty} y_{n} R^{n} e^{i n t}$. (Trivially, much more could be said about $u_{R}^{a}$ and the series, but nothing more is needed.)

Now let $Y \subset X^{\prime}$ as in 2.2 and $1 / q+1 / p=1 .\left(u_{R}^{a}\right)_{R<1}$ is a net in $L^{p}(Y) \subset$ $L_{Y}^{p}\left(X^{\prime}, X\right) \subset L^{p}\left(X^{\prime}, X\right)=L^{q}(X)^{\prime}$ (Theorem 2.1), bounded by $A_{p}\|u\|_{p}$. By the Banach-Alaoglu theorem, there is a $\sigma\left(L^{p}\left(X^{\prime}, X\right), L^{q}(X)\right)$-accumulation point $\varphi$ of $\left(u_{R}^{a}\right)_{R<1}$ with $\|\varphi\|_{p} \leq A_{p}\|u\|_{p}$. I claim that $\varphi \sim \sum_{n=0}^{\infty} y_{n} e^{i n t}$ : For any $\psi \in L^{q}(X)$, $\int_{T}\langle\psi, \varphi\rangle d \lambda$ is an accumulation point (as $\left.R \rightarrow 1\right)$ of $\int_{T}\left\langle\psi, u_{R}^{a}\right\rangle d \lambda$. Fix $x \in X$ and $n \in \mathbf{Z}$ and take $\psi(t):=x e^{-i n t}$. Then $\langle x, \hat{\varphi}(n)\rangle$ is an accumulation point (as $R \rightarrow 1)$ of $\left\langle x, \widehat{u_{R}^{a}}(n)\right\rangle=\left\langle x, R^{n} y_{n} \chi_{\mathbf{N}_{0}}(n)\right\rangle \stackrel{R \rightarrow 1}{\longrightarrow}\left\langle x, y_{n} \chi_{\mathbf{N}_{0}}(n)\right\rangle$. Thus $\langle x, \hat{\varphi}(n)\rangle=$ $\left\langle x, y_{n} \chi_{\mathbf{N}_{0}}(n)\right\rangle$ for all $x \in X, n \in \mathbf{Z}$ and the claim is proved. It implies in particular that $\varphi \in L_{Y, a}^{p}\left(X^{\prime}, X\right)$, so that $P[\varphi] \in H^{p}(Y),\|P[\varphi]\|_{p}=\|\varphi\|_{p} \leq A_{p}\|u\|_{p}$. But $P[\varphi](z)=\sum_{n=0}^{\infty} y_{n} z^{n}$, i.e., $P[\varphi]=u^{a} \in H^{p}(Y),\left\|u^{a}\right\|_{p} \leq A_{p}\|u\|_{p}$.

A note for expert readers: if $Y$ satisfies $\left(1^{\circ}\right)(\mathrm{a})$, then, as noted in the introduction, the $Y$-valued Hilbert transform is $\|\cdot\|_{p}$-bounded which implies that $Y$ is superreflexive (e.g. $[\mathbf{1}, \mathbf{1 1}])$. In particular, $Y$ has the Radon-Nikodym property. But then, by the work of Bukhvalov and Danilevich [4], Poisson integral defines isometric isomorphisms $L^{p}(Y) \cong h^{p}(Y)$ and $\mathbf{H}^{p}(Y) \cong H^{p}(Y)$. Since analytic projection commutes with the Poisson isomorphism (see 2.5$),\left(1^{\circ}\right)(\mathrm{b})$ is now tautologically the same as $\left(1^{\circ}\right)(\mathrm{a})$. On the other hand, the self-contained, direct proof given above seems to me to be the natural one, whereas the approach just sketched would be similar to proving there are infinitely many primes by using the prime number theorem.

3.2 Proof of $\left(2^{\circ}\right)(\mathrm{a}) \Rightarrow\left(1^{\circ}\right)(\mathrm{a})$ and $\left(2^{\circ}\right)(\mathrm{b}) \Rightarrow\left(1^{\circ}\right)(\mathrm{a})$. See $\S 1$ for the idea (namely, Rudin's) of proof. In particular, as is stated there already, $\left(2^{\circ}\right)(\mathrm{a}) \Rightarrow\left(1^{\circ}\right)$ (a) could be proved by a verbatim copy of $[\mathbf{1 5}, \mathrm{p}$. 154]. This is because, as in the scalar case, rotation in the argument of a Bochner integrable function $\varphi \in L^{p}(Y)$ is a continuous map $T \rightarrow L^{p}(Y)(1 \leq p<\infty,[14,3.8])$. Let $Y \subset X^{\prime}$. Then condition $\left(2^{\circ}\right)(\mathrm{b})$, carried over to the circle $T$ by means of the Poisson isomorphism 2.3, reads

$\left(2^{\circ}\right)\left(\mathrm{b}^{\prime}\right) L_{Y, a}^{p}\left(X^{\prime}, X\right)$ is complemented in $L_{Y}^{p}\left(X^{\prime}, X\right)$,

and for any only Gel fand integrable function $\varphi \in L^{p}\left(X^{\prime}, X\right)$ the above mentioned continuity property fails in general (consider, e.g., $\left.\varphi: T \rightarrow l^{\infty}, \varphi(t):=\left(e^{i n t}\right)_{n \in \mathrm{N}}\right)$. 
Nevertheless, it enters the proof of $\left(2^{\circ}\right)(b) \Rightarrow\left(1^{\circ}\right)(a)$, too, in a more hidden fashion, though.

The two implications will now be proved simultaneously. Either of the conditions $\left(2^{\circ}\right)(\mathrm{a})$ and $\left(2^{\circ}\right)(\mathrm{b})$ (in the form $\left(2^{\circ}\right)\left(\mathrm{b}^{\prime}\right)$ ) imply the

Hypothesis. $\exists S: L^{p}(Y) \rightarrow L_{Y, a}^{p}\left(X^{\prime}, X\right)$ bounded linear such that $S \mid \mathbf{H}^{p}(Y)=$ $i d_{\mathbf{H}^{p}(Y)}$.

Assertion. Analytic projection is bounded in $\|\cdot\|_{p}$ on the $Y$-valued trigonometric polynomials (as remarked in 2.5 , this is equivalent to $\left(1^{\circ}\right)(\mathrm{a})$ ).

I need the following

LEMMA. Let $1<p \leq \infty, 1 / p+1 / q=1, m \in \mathbf{Z}$ and $F_{m}: L^{p}\left(X^{\prime}, X\right) \rightarrow X^{\prime}$, $f \mapsto \hat{f}(m)$. Suppose that $\varphi: T \rightarrow L^{p}\left(X^{\prime}, X\right)=L^{q}(X)^{\prime}$ is scalarly integrable (w.r.t. $\left.L^{q}(X)\right)$. Then $F_{m} \circ \varphi: T \rightarrow X^{\prime}$ is scalarly integrable (w.r.t. $\left.X\right)$ and $F_{m}\left[(G) \int_{T} \varphi d \lambda\right]=(G) \int_{T} F_{m} \circ \varphi d \lambda$.

From now on, $\int_{T}$ will be abbreviated to $\int$, and the duality between $L^{q}(X)$ and $L^{p}\left(X^{\prime}, X\right)$ will often be denoted by $\langle\cdot, \cdot\rangle$, too.

PROOF OF LEMMA. $F_{m} \circ \varphi: T \rightarrow X^{\prime}$ is scalarly integrable: Let $x \in X$. Now

$$
\begin{aligned}
\left\langle x, F_{m}[\varphi(\vartheta)]\right\rangle & =\left\langle x,(G) \int e^{-i m t} \varphi(\vartheta)(t) \frac{d t}{2 \pi}\right\rangle=\int\left\langle x e^{-i m t}, \varphi(\vartheta)(t)\right\rangle \frac{d t}{2 \pi} \\
& =\int\langle g(t), \varphi(\vartheta)(t)\rangle \frac{d t}{2 \pi}=\langle g, \varphi(\vartheta)\rangle
\end{aligned}
$$

(where $g \in L^{q}(X), g(t):=x e^{-i m t}$ ). This is integrable in $\vartheta$, just by the assumption on $\varphi$. Asserted equality: Fix $x \in X$. By definition of the $\mathrm{Gel}^{\prime}$ fand integral, we have

$$
\left\langle x,(G) \int F_{m} \circ \varphi d \lambda\right\rangle=\int\left\langle x, F_{m}[\varphi(\vartheta)]\right\rangle \frac{d \vartheta}{2 \pi}=\int\langle g, \varphi(\vartheta)\rangle \frac{d \vartheta}{2 \pi},
$$

where $g$ is as above. On the other hand, with $\Phi:=(G) \int \varphi d \lambda \in L^{p}\left(X^{\prime}, X\right)=$ $L^{q}(X)^{\prime}$, we have

$$
\begin{aligned}
\left\langle x, F_{m}\left[(G) \int \varphi d \lambda\right]\right\rangle & =\left\langle x, F_{m}[\Phi]\right\rangle=\left\langle x,(G) \int e^{-i m t} \Phi(t) \frac{d t}{2 \pi}\right\rangle \\
& =\int\left\langle x e^{-i m t}, \Phi(t)\right\rangle \frac{d t}{2 \pi}=\langle g, \Phi\rangle \\
& =\left\langle g,(G) \int \varphi d \lambda\right\rangle=\int\langle g, \varphi\rangle d \lambda . \quad \text { Q.E.D. }
\end{aligned}
$$

(A good exercise in unravelling definitions.)

PROOF OF ASSERTION ASSUMING HYPOTHESIS. Let $1<p<\infty, 1 / p+1 / q=1$. For $\vartheta \in T, f \in L_{Y}^{p}\left(X^{\prime}, X\right)$ define $R_{\vartheta} f(t):=f(\vartheta+t)$. Clearly $R_{\vartheta} f \in L_{Y}^{p}\left(X^{\prime}, X\right)$, $\left\|R_{\vartheta} f\right\|_{p}=\|f\|_{p}, f \in L^{p}(Y)$ implies $R_{\vartheta} f \in L^{p}(Y)$, and $f \in L_{Y, a}^{p}\left(X^{\prime}, X\right)$ implies $R_{\vartheta} f \in L_{Y, a}^{p}\left(X^{\prime}, X\right)$. Now let $f \in L^{p}(Y)$ be a trigonometric polynomial.

Claim 1. $\varphi: T \rightarrow L^{p}\left(X^{\prime}, X\right)=L^{q}(X)^{\prime}, \vartheta \mapsto R_{-\vartheta} S R_{\vartheta} f$ is scalarly integrable.

ProOF. W.l.o.g., $f$ is a monomial $f(t)=y e^{i n t}$ with fixed $y \in Y, n \in \mathbf{Z}$. Then, for $\vartheta \in T, R_{\vartheta} f(t)=y e^{i n(\vartheta+t)}=e^{i n \vartheta} f(t)$, i.e., $R_{\vartheta} f=e^{i n \vartheta} f$, so that $\varphi(\vartheta)=\left(R_{-\vartheta} S\right)\left(R_{\vartheta} f\right)=e^{i n \vartheta} R_{-\vartheta} S f=e^{i n \vartheta} R_{-\vartheta} g$, putting $g:=S f$. The claim says 
that for any $h \in L^{q}(X)$, the scalar function $\vartheta \mapsto\langle h, \varphi(\vartheta)\rangle$ is integrable. Now

$$
\begin{aligned}
\langle h, \varphi(\vartheta)\rangle & =\int\langle h(t), \varphi(\vartheta)(t)\rangle \frac{d t}{2 \pi} \\
& =\int\left\langle h(t), e^{i n \vartheta}\left(R_{-\vartheta} g\right)(t)\right\rangle \frac{d t}{2 \pi} \\
& =e^{i n \vartheta} \int\langle h(t), g(t-\vartheta)\rangle \frac{d t}{2 \pi} \\
& =e^{i n \vartheta} \int\langle h(t+\vartheta), g(t)\rangle \frac{d t}{2 \pi} \\
& =e^{i n \vartheta} \int\left\langle R_{\vartheta} h(t), g(t)\right\rangle \frac{d t}{2 \pi} \\
& =e^{i n \vartheta}\left\langle R_{\vartheta} h, g\right\rangle
\end{aligned}
$$

$\left(R_{\vartheta}\right.$ now also operates $\left.L^{q}(X) \rightarrow L^{q}(X)\right)$. This calculation reduces matters to the Bochner integrable case: For $h \in L^{q}(X)$, the map $T \rightarrow L^{q}(X), \vartheta \mapsto R_{\vartheta} h$, is even continuous $[\mathbf{1 4}, 3.8]$, and so is the function under consideration $\vartheta \mapsto\langle h, \varphi(\vartheta)\rangle=$ $e^{i n \vartheta}\left\langle R_{\vartheta} h, g\right\rangle$. Claim 1 is proved.

For any trigonometric polynomial $f \in L^{p}(Y)$ we can thus define

$$
\tilde{S} f:=(G) \int R_{-\vartheta} S R_{\vartheta} f \frac{d \vartheta}{2 \pi} \in L^{p}\left(X^{\prime}, X\right)
$$

Claim 2. $\tilde{S}:\left\{f \in L^{p}(Y): f\right.$ is a trigonometric polynomial $\} \rightarrow L^{p}\left(X^{\prime}, X\right)$ is bounded.

PrOOF. Since $L^{p}\left(X^{\prime}, X\right)=L^{q}(X)^{\prime}$, we have for any trigonometric polynomial $f \in L^{p}(Y)$

$$
\begin{aligned}
\|\tilde{S} f\|_{p} & =\sup \left\{|\langle h, \tilde{S} f\rangle|: h \in L^{q}(X),\|h\|_{q} \leq 1\right\} \\
& =\sup \left\{\left|\int\left\langle h, R_{-\vartheta} S R_{\vartheta} f\right\rangle \frac{d \vartheta}{2 \pi}\right|: h \in L^{q}(X),\|h\|_{q} \leq 1\right\} \\
& \leq \sup \left\{\int\left|\left\langle h, R_{-\vartheta} S R_{\vartheta} f\right\rangle\right| \frac{d \vartheta}{2 \pi}: h \in L^{q}(X),\|h\|_{q} \leq 1\right\} \\
& \leq\|S\|\|f\|_{p}
\end{aligned}
$$

the last inequality because

$$
\left|\left\langle h, R_{-\vartheta} S R_{\vartheta} f\right\rangle\right| \leq\|h\|_{q}\left\|R_{-\vartheta} S R_{\vartheta} f\right\|_{p} \leq\|S\|\|f\|_{p}
$$

Claim 3. $\tilde{S}$ is the analytic projection.

PROOF. It suffices to show this for monomials $f(t)=y e^{i n \vartheta} ; y \in Y, n \in \mathbf{Z}$ fixed.

Case 1. $n \geq 0$. Then $f \in \mathbf{H}^{p}(Y)$, whence $R_{\vartheta} f \in \mathbf{H}^{p}(Y)$ and $S R_{\vartheta} f=R_{\vartheta} f$, by assumption on $S$. But then $R_{-\vartheta} S R_{\vartheta} f=f$, independently of $\vartheta$, so that $\tilde{S} f=f=$ $f^{a}$.

Case 2. $n<0$. Then $f^{a}=0$, so we must show $\tilde{S} f=0$ (in $L^{p}\left(X^{\prime}, X\right)$ ). To this end, the lemma will be used to prove that all Fourier coefficients of $\tilde{S} f$ vanish (then we conclude $\langle x, \tilde{S} f\rangle^{\wedge}(m)=\left\langle x,(\tilde{S} f)^{\wedge}(m)\right\rangle=0$, all $x \in X, m \in \mathbf{Z}$, whence $\langle x, \tilde{S} f\rangle=0$, all $x \in X$, which is the assertion). So let $m \in \mathbf{Z}$ be fixed and 
$F_{m}: L^{p}\left(X^{\prime}, X\right) \rightarrow X^{\prime}, f \mapsto \hat{f}(m)$ as in the lemma. The latter makes $F_{m} \circ \varphi$ scalarly integrable and gives

$$
F_{m}[\tilde{S} f]=F_{m}\left[(G) \int \varphi(\vartheta) \frac{d \vartheta}{2 \pi}\right]=(G) \int F_{m}[\varphi(\vartheta)] \frac{d \vartheta}{2 \pi}
$$

As computed in the proof of claim 1 , for $f(t)=y e^{i n t}$, we have $\varphi(\vartheta)=e^{i n \vartheta} R_{-\vartheta} g$, putting $g:=S f$. Thus

$$
\begin{aligned}
F_{m}[\varphi(\vartheta)] & =e^{i n \vartheta} F_{m}\left[R_{-\vartheta} g\right] \\
& =e^{i n \vartheta}(G) \int e^{-i m t}\left(R_{-\vartheta} g\right)(t) \frac{d t}{2 \pi} \\
& =e^{i n \vartheta}(G) \int e^{-i m t} g(t-\vartheta) \frac{d t}{2 \pi} \\
& =e^{i(n-m) \vartheta}(G) \int e^{-i m t} g(t) \frac{d t}{2 \pi} \\
& =e^{i(n-m) \vartheta} \hat{g}(m) .
\end{aligned}
$$

If $m<0$, this is already zero for all $\vartheta$, since $g=S f \in L_{Y, a}^{p}\left(X^{\prime}, X\right)$; so much the more is $F_{m}[\tilde{S} f]=\int F_{m}[\varphi(\vartheta)] \frac{d \vartheta}{2 \pi}=0$. If $m \geq 0$, the above yields $F_{m}[\tilde{S} f]=$ $(2 \pi)^{-1} \int e^{i(n-m) \vartheta} d \vartheta \hat{g}(m)=0$, since $n<0 \leq m$. Q.E.D.

The following remark, which is trivial modulo scalar theory, completes the theorem as regards the extreme exponents $p=1, \infty$. In its formulation, $H^{1}(Y)$ is identified with $L_{Y, a}^{1}\left(X^{\prime}, X\right)$ (see Theorem 2.4).

3.3 REMARK. Suppose $0 \neq Y \subset X^{\prime}$. The following inclusions are not complemented:

$$
\begin{gathered}
\mathbf{H}^{1}(Y) \subset L^{1}(Y), \quad H^{1}(Y) \subset L_{Y}^{1}\left(X^{\prime}, X\right) \quad\left(\text { whence also } H^{1}(Y) \subset h^{1}(Y)\right), \\
\mathbf{H}^{\infty}(Y) \subset L^{\infty}(Y), \quad H^{\infty}(Y) \subset h^{\infty}(Y) .
\end{gathered}
$$

ProOF. For $Y=\mathbf{C}$, the assertions are in [15, p. $154 \mathrm{f}$.$] (Newman's theo-$ rem). Choose $x \in X, y \in Y$ with $\langle x, y\rangle=1$. Suppose, e.g., $S: L^{1}(Y) \rightarrow \mathbf{H}^{1}(Y)$ were a projection, then $S_{\mathbf{C}}: L^{1} \rightarrow H^{1}, f \mapsto\langle x, S[f y]\rangle$ would be a projection, too; contradiction. The other assertions are proved in the same way.

For $\mathbf{H}^{1}(Y), \mathbf{H}^{\infty}(Y)$ the remark is due to Bukhvalov [3, Theorem 3.9] who observed that these assertions can be proved "just as in the scalar case". On the other hand, the proof of the main theorem works equally well for $p=\infty$ (curiously, not for $p=1$ !) giving the remark for $\mathbf{H}^{\infty}(Y), H^{\infty}(Y)$.

\section{REFERENCES}

1. J. Bourgain, Some remarks on Banach spaces in which martingale difference sequences are unconditional, Ark. Mat. 21 (1983), 163-168.

2. A. V. Bukhvalov, On an analytic representation of operators with abstract norm, Soviet Math. Dokl. 14 (1973), 197-201.

3. $\ldots$, Hardy spaces of vector-valued functions, J. Soviet Math. 16 (1981), 1051-1059.

4. A. V. Bukhvalov and A. A. Danilevich, Boundary properties of analytic and harmonic functions with values in Banach space, Math. Notes Acad. Sci. USSR 31 (1982), 104-110.

5. D. L. Burkholder, A geometric condition that implies the existence of certain singular integrals of Banach-space-valued functions, Proc. Conf. Harmonic Analysis in Honour of A. Zygmund, Univ. of Chicago, 1981, 1983, pp. 270-286. 
6. - Martingales and Fourier analysis in Banach spaces, Probability and Analysis (Varenna, (Como), 1985), (G. Letta and M. Pratelli, eds.), Lecture Notes in Math., vol. 1206, SpringerVerlag, Berlin, Heidelberg and New York, 1986, pp. 61-108.

7. J. Diestel and J. J. Uhl, Jr., Vector measures, Math. Surveys, No. 15, Amer. Math. Soc., Providence, R. I., 1977.

8. N. Dinculeanu, Vector measures, VEB Deutscher Verlag der Wissenschaften, Berlin, 1966 (Hochschulbücher für Math. 64).

9. C. Grossetête, Sur certaines classes de fonctions harmoniques dans le disque à valeur dans un espace vectoriel topologique localement convexe, C. R. Acad. Sci. Paris 273 (1971), 1048-1051.

10. __ Classes de Hardy et de Nevanlinna pour les fonctions holomorphes à valeurs vectorielles, C. R. Acad. Sci. Paris 274 (1972), 251-253.

11. J. A. Gutierrez and H. E. Lacey, On the Hilbert transform for Banach space valued functions, Martingale Theory in Harmonic Analysis and Banach Spaces (Cleveland, 1981), Lecture Notes in Math., vol. 939, Springer-Verlag, Berlin, Heidelberg and New York, 1982, pp. 7380.

12. M. Heins, Vector-valued harmonic functions, Functions, Series, Operators, Colloq. Math. Soc. J. Bolyai 35 (1980), 621-632.

13. W. Hensgen, Hardy-Räume vektorwertiger Funktionen, Thesis, Munich, 1986.

14. E. Hille and R. S. Phillips, Functional analysis and semi-groups, Amer. Math. Soc. Colloq. Publ., vol. 23, Amer. Math. Soc., Providence, R.I., 1957.

15. K. Hoffmann, Banach Spaces of Analytic Functions, Prentice Hall, Englewood Cliffs, N.J., 1962.

16. A. Ionescu-Tulcea and C. Ionescu-Tulcea, Topics in the theory of lifting, Springer-Verlag, Berlin, Heidelberg and New York, 1969 (Ergebnisse... 48).

17. W. Kaballo, On Fredholm operator valued $H^{p}$-functions, Proc. Toeplitz Mem. Conf., Tel Aviv, Birkhäuser, 1982, pp. 313-319.

18. J. Lindenstrauss and L. Tzafriri, Classical Banach spaces. II, Springer-Verlag, Berlin, Heidelberg and New York, 1979 (Ergebnisse... 97).

19. W. J. Ricker, Characterization of Poisson integrals of vector-valued functions and measures on the unit circle, Hokkaido Math. J. 16 (1987), $29-42$.

20. R. Ryan, Boundary values of analytic vector-valued functions, Indag. Math. 65 (1962), 558-572.

21. _ The F. and M. Riesz theorem for vector measures, Indag. Math. 66 (1963), 408-412.

NATURWISSENSCHAFTLICHE FAKULtät I, MATHEMATIK, UNIVERSität REGENSBURG, UNIVERSITÄTSSTR. 31, 8400 REgENSBURG, FEDERAL REPUBLIC OF GERMANY 